\title{
Genome-scale fitness profile of Caulobacter crescentus grown in natural freshwater
}

\author{
Kristy L. Hentchel ${ }^{1}$ - Leila M. Reyes Ruiz ${ }^{1}$ Patrick D. Curtis ${ }^{2}$ - Aretha Fiebig ${ }^{1} \cdot$ Maureen L. Coleman $\mathbb{1}^{3}$ • \\ Sean Crosson (1) ${ }^{1}$
}

Received: 3 May 2018 / Revised: 18 September 2018 / Accepted: 21 September 2018 / Published online: 8 October 2018

(c) International Society for Microbial Ecology 2018

\begin{abstract}
Bacterial genomes evolve in complex ecosystems and are best understood in this natural context, but replicating such conditions in the lab is challenging. We used transposon sequencing to define the fitness consequences of gene disruption in the bacterium Caulobacter crescentus grown in natural freshwater, compared with axenic growth in common laboratory media. Gene disruptions in amino-acid and nucleotide sugar biosynthesis pathways and in metabolic substrate transport machinery impaired fitness in both lake water and defined minimal medium relative to complex peptone broth. Fitness in lake water was enhanced by insertions in genes required for flagellum biosynthesis and reduced by insertions in genes involved in biosynthesis of the holdfast surface adhesin. We further uncovered numerous hypothetical and uncharacterized genes for which disruption impaired fitness in lake water, defined minimal medium, or both. At the genome scale, the fitness profile of mutants cultivated in lake water was more similar to that in complex peptone broth than in defined minimal medium. Microfiltration of lake water did not significantly affect the terminal cell density or the fitness profile of the transposon mutant pool, suggesting that Caulobacter does not strongly interact with other microbes in this ecosystem on the measured timescale. Fitness of select mutants with defects in cell surface biosynthesis and environmental sensing were significantly more variable across days in lake water than in defined medium, presumably owing to day-to-day heterogeneity in the lake environment. This study reveals genetic interactions between Caulobacter and a natural freshwater environment, and provides a new avenue to study gene function in complex ecosystems.
\end{abstract}

Electronic supplementary material The online version of this article (https://doi.org/10.1038/s41396-018-0295-6) contains supplementary material, which is available to authorized users.

Aretha Fiebig

aretha@uchicago.edu

$\triangle$ Maureen L. Coleman

mlcoleman@uchicago.edu

$\triangle$ Sean Crosson

scrosson@uchicago.edu

1 Department of Biochemistry and Molecular Biology, University of Chicago, Chicago, IL 60637, USA

2 Department of Biology, University of Mississippi, University, MS 38677, USA

3 Department of the Geophysical Sciences, University of Chicago, Chicago, IL 60637, USA

\section{Introduction}

Environments inhabited by microbial cells have significant microscale heterogeneity. This is well recognized in biofilms, soils, and host-associated habitats [1-3]. Free-living aquatic bacteria often encounter chemical gradients that can appear as ephemeral patches, arising from algal exudates, sinking particles, or lysis events $[4,5]$ and they may have to cope with prolonged periods of nutrient scarcity. In addition, these bacteria face interspecies interactions, protistan predators, and viruses, as well as fluctuations in physical conditions such as temperature and light. These biotic and abiotic factors have driven myriad adaptations that enable survival and reproduction in natural environments.

In contrast with this natural complexity, studies on microbial physiology and gene function have traditionally relied on simplified experimental conditions. Thus, it is not surprising that a large fraction of bacterial genes remain uncharacterized. Recently developed transposon sequencing (Tn-Seq) approaches [6] now enable rapid phenotypic 
Table 1 Analysis of Lake Michigan water used for barcoded Tn-Himar fitness experiments

\begin{tabular}{|c|c|c|c|c|}
\hline Water collection ${ }^{\mathrm{a}}$ & 30 Nov. & 6 Dec. & 9 Dec. & 12 Dec. \\
\hline Date of experiment & 2 Dec. & 6 Dec. & 9 Dec. & 12 Dec. \\
\hline Water temperature $\left({ }^{\circ} \mathrm{C}\right)$ & 7.5 & 7 & 3 & 2 \\
\hline Air temperature $\left({ }^{\circ} \mathrm{C}\right)$ & 7.8 & 4.4 & -3.3 & -7.2 \\
\hline $\mathrm{pH}$ & 5.8 & 5.8 & 5.8 & 5.8 \\
\hline Phosphate & $5 \mathrm{ppm}$ & $5 \mathrm{ppm}$ & $5 \mathrm{ppm}$ & $5 \mathrm{ppm}$ \\
\hline Nitrate/nitrite ${ }^{\mathrm{b}}$ & Undetectable & Undetectable & Undetectable & Undetectable \\
\hline
\end{tabular}

${ }^{a}$ Water collection occurred in 2016 at Promontory Point, Hyde Park, Chicago, Illinois, USA

${ }^{b}$ Limit of detection: nitrite $0.15 \mathrm{mg} \mathrm{L}^{-1}$, nitrate $1 \mathrm{mg} \mathrm{L}^{-1}$ assessment of thousands to millions of distinct mutant strains, and these methods have been used to interrogate gene function in a variety of in vitro and host-associated conditions [7, 8]. More recently, transposon mutagenesis approaches in which each transposon carries a unique 20-bp barcode sequence have been developed [9]; each insertion site is associated with a short barcode, and the abundance of all mutant strains in the pool can be assessed by simple amplicon sequencing.

Here, we used a barcoded Tn-Seq approach to identify genes affecting fitness in Caulobacter crescentus strain CB15, cultivated in natural freshwater from Lake Michigan, Illinois, USA. As a well-characterized and genetically tractable bacterium originally isolated from a pond in California in 1960 [10], this strain is well suited for this study. Briefly, C. crescentus is among a group of dimorphic prosthecate (i.e., stalked) bacteria that attach to surfaces, often forming epibiotic interactions with algae and plant material [11]. More broadly, members of the genus Caulobacter are common in soil ecosystems, where they likely play an important role in plant matter decomposition [12]. In aquatic systems, Caulobacter interactions with substrates contribute to biopolymer mineralization, and have been proposed to enhance productivity of aquatic ecosystems $[11,13]$. However, C. crescentus (hereafter referred to simply as Caulobacter) is typically grown in an artificial medium consisting of dilute peptone and yeast extract (PYE) or in a defined medium consisting of mineral salts and a single carbon source such as xylose (M2X) [14], neither of which adequately represents natural freshwater. PYE is replete with peptides, amino acids, and a range of carbon sources, whereas M2X requires Caulobacter to synthesize all cellular building blocks from salts and a simple sugar. Natural freshwaters, by contrast, contain an undefined, complex mixture of organic and inorganic nutrient sources [15]. In many freshwater systems, essential nutrients including phosphorus and labile carbon do not accumulate to high concentrations $[16,17]$. We predicted that genes that are dispensable in PYE [18] or M2X medium would be important for fitness in complex natural freshwater, and that these genes would offer insights into Caulobacter physiology in a bona fide freshwater system.

\section{Materials and methods}

\section{Bacterial strains and primers}

Strains and primers used in this study are listed in Table S1. All primers were synthesized by Integrated DNA Technologies (Coralville, IA, USA).

\section{Growth media}

C. crescentus strain CB15 [10] was grown in PYE medium [0.2\% peptone (Fisher Scientific), 0.1\% yeast extract (Fisher Scientific), $0.5 \mathrm{mM} \mathrm{MgSO}_{4}, 0.5 \mathrm{mM} \mathrm{CaCl}$ ] or $\mathrm{M} 2 \mathrm{X}$ minimal defined medium $\left[6.1 \mathrm{mM} \mathrm{Na}_{2} \mathrm{HPO}_{4}, 3.9 \mathrm{mM}\right.$ $\mathrm{KH}_{2} \mathrm{PO}_{4}, 9.3 \mathrm{mM} \mathrm{NH} 4 \mathrm{Cl}, 0.5 \mathrm{mM} \mathrm{MgSO}, 10 \mu \mathrm{M} \mathrm{FeSO}_{4}$ (EDTA chelate; Sigma Chemical Co.), $0.25 \mathrm{mM} \mathrm{CaCl}_{2}$ ] supplemented with $0.15 \%$ xylose [14]. Escherichia coli strains were grown in lysogeny broth (LB) (1\% peptone, $0.5 \%$ yeast extract, $0.5 \% \mathrm{NaCl})$. Solid growth media included $1.5 \%$ agar.

\section{Lake water collection}

Water from Lake Michigan was collected at Promontory Point, Chicago, Illinois, USA (latitude: 41.794, longitude: -87.579), on four dates in 2016 (30 Nov, 6 Dec, 9 Dec, and $12 \mathrm{Dec})$. We measured water temperature, phosphate, and nitrate/nitrite level (Aquacheck Water Quality Test Strips; Hach), and $\mathrm{pH}$ (pH indicator strips, Fisher Scientific) at the time of collection (Table 1). Lake water was filtered using Nalgene $^{\mathrm{TM}}$ Rapid-Flow ${ }^{\mathrm{TM}}$ Sterile Disposable $0.1 \mu \mathrm{m}$ Filter Units with PES Membrane (Thermo Scientific).

\section{Measurement of Caulobacter growth in lake water}

Colonies of Caulobacter were inoculated into $2 \mathrm{~mL}$ of PYE in glass culture tubes $(13 \times 100 \mathrm{~mm})$ and grown overnight at $30{ }^{\circ} \mathrm{C}$ with shaking at $200 \mathrm{rpm}$, for a total of five biological replicates. At saturation, $1 \mathrm{~mL}$ of culture was centrifuged at $8000 \times g$ and washed twice in $1 \mathrm{~mL}$ of filtered lake water. The washed pellet was resuspended in filtered lake water to a final $\mathrm{OD}_{660}$ of 0.1 , and $0.5 \mu \mathrm{L}$ (approximately $0.5-1 \times 10^{5}$ 
cells) was inoculated into $5 \mathrm{~mL}$ of filtered lake water in a glass culture tube $(20 \mathrm{~mm} \times 150 \mathrm{~mm})$ in technical duplicate. Cultures were grown at $30{ }^{\circ} \mathrm{C}$ with shaking at $200 \mathrm{rpm}$. To monitor growth, $20 \mu \mathrm{L}$ of culture was removed at various time points, serially diluted, and titered onto PYE agar plates, which were incubated at $30^{\circ} \mathrm{C}$ for 2 days. Growth was monitored by enumeration of colony-forming units (CFUs).

\section{Construction of barcoded Tn-Himar mutant library}

The recipient strain (Caulobacter) was grown overnight in $2 \mathrm{~mL}$ of PYE at $30^{\circ} \mathrm{C}$ with shaking at $200 \mathrm{rpm}$. This starter culture was used to inoculate $20 \mathrm{~mL}$ of PYE and grown at $30{ }^{\circ} \mathrm{C}$ overnight with shaking at $200 \mathrm{rpm}$ until saturated. The donor $E$. coli strain (APA752, gift from Adam Deutschbauer, University of California-Berkeley, USA), carrying the pKMW3 (kanamycin resistant) Himar transposon vector library [9], was inoculated into $20 \mathrm{~mL}$ of LB containing kanamycin $\left(30 \mu \mathrm{g} \mathrm{mL}^{-1}\right)$ and diaminopimelate (DAP; $300 \mu \mathrm{M}$ ) and grown overnight at $37^{\circ} \mathrm{C}$ with shaking at $200 \mathrm{rpm}$; the E. coli Himar donor strain is a DAP auxotroph, and thus requires addition to the medium. To conjugate the barcoded transposon pool into Caulobacter, the recipient strain and donor strains were each centrifuged at $8000 \times g$ for $2 \mathrm{~min}$ and resuspended in a total volume of $500 \mu \mathrm{L}$ of PYE medium. The cultures were combined at a 10:1 ratio of recipient to donor and mixed by gentle pipetting. The mixed culture was centrifuged again at $8000 \times g$, and the supernatant decanted. The cells were resuspended in $30 \mu \mathrm{L}$ of PYE, spotted onto a PYE agar plate containing diaminopimelate $(300 \mu \mathrm{M})$, and incubated overnight at $30{ }^{\circ} \mathrm{C}$. After growth, the mating spot was scraped from the plate and resuspended in $6.5 \mathrm{~mL}$ of PYE. This suspension was spread evenly $(500 \mu \mathrm{L}$ per plate) over 14 large $(150 \times 15 \mathrm{~mm})$ PYE agar plates containing $25 \mu \mathrm{g} \mathrm{mL}^{-1}$ kanamycin and incubated for approximately 3 days at $30^{\circ} \mathrm{C}$. Cells were harvested from all the plates and inoculated into $400 \mathrm{~mL}$ of PYE containing $5 \mu \mathrm{g} \mathrm{mL}^{-1}$ kanamycin. This cell mixture was grown at $30^{\circ} \mathrm{C}$ with shaking at $200 \mathrm{rpm}$ for three doublings. Cells were centrifuged at $8000 \times g$, resuspended in $70 \mathrm{~mL}$ of PYE containing $15 \%$ glycerol, and stored as $1 \mathrm{~mL}$ aliquots at $-80{ }^{\circ} \mathrm{C}$.

\section{Mapping of the sites of Tn-Himar insertion in the Caulobacter BarSeq library}

Genomic DNA was extracted using guanidium thiocyanate as previously described [19]. The DNA was sheared ( 300 bp fragments), cleaned with a standard bead protocol, endrepaired and A-tailed, and a custom double-stranded $\mathrm{Y}$ adapter was ligated. The custom adapter was prepared by annealing Mod2_TS_Univ and Mod2_TruSeq (Table S1) as described [9]. The sheared fragments containing transposons were enriched by PCR using the primers Nspacer_BarSeq_pHIMAR and P7_MOD_TS_index1 using GoTaq $^{\circledR}$ Green Master Mix according to the manufacturer's protocol in a $100-\mu \mathrm{L}$ volume with the following cycling conditions: $94{ }^{\circ} \mathrm{C}$ for $2 \mathrm{~min}, 25$ cycles at $94{ }^{\circ} \mathrm{C}$ for $30 \mathrm{~s}, 65^{\circ}$ $\mathrm{C}$ for $20 \mathrm{~s}$, and $72{ }^{\circ} \mathrm{C}$ for $30 \mathrm{~s}$, followed by a final extension at $72{ }^{\circ} \mathrm{C}$ for $10 \mathrm{~min}$. After a second bead cleanup, the Caulobacter library was sequenced using a standard Illumina sequencing primer on an Illumina HiSeq2500 at the University of Chicago Genomics Facility with a 150-bp single-end read. The locations of Himar transposon insertions were aligned and mapped using BLAT [20], and unique barcode sequences were associated with their corresponding genome insertion location using a custom Perl script (MapTnSeq.pl). Sets of barcodes that consistently mapped to one location in the genome were identified using a custom Perl script (DesignRandomPool.pl). This ensures that each unique barcode is properly assigned to a single insertion site. These scripts have been described by Wetmore and colleagues [9] and are available at https:// bitbucket.org/berkeleylab/feba. For an outline of this workflow, see Figure S1. For all analyses, reads were mapped to the $C$. crescentus NA1000 genome (accession CP001340) [21], which is more comprehensively annotated [22] than the highly related CB15 parent strain.

\section{Cultivation of the Tn-Himar library}

An aliquot of the Caulobacter library $(2 \mathrm{~mL})$ from a glycerol stock was inoculated into $18 \mathrm{~mL}$ of PYE, split into two tubes $(20 \times 150 \mathrm{~mm})$ with $10 \mathrm{~mL}$ each, and grown in a cell culture roller drum (Fisher Scientific) at $30^{\circ} \mathrm{C}$ for $4 \mathrm{~h}$. The tubes were then moved to a $30^{\circ} \mathrm{C}$ incubator with shaking at $200 \mathrm{rpm}$ for an additional $2 \mathrm{~h}$. Cultures were combined and centrifuged for $20 \mathrm{~min}$ at $3000 \times g$ at $4{ }^{\circ} \mathrm{C}$. The cell pellet was resuspended and washed in $10 \mathrm{~mL}$ of filtered lake water, and centrifuged again at $3000 \times g$ for $20 \mathrm{~min}$ at $4{ }^{\circ} \mathrm{C}$. The resulting pellet was resuspended in $5 \mathrm{~mL}$ of filtered lake water, and the $\mathrm{OD}_{660}$ measured. Flasks containing filtered or unfiltered lake water $(7.5 \mathrm{~L}$ total volume per condition, divided over three flasks) were inoculated with the washed library with the aim of an initial starting concentration of approximately $2.5 \times 10^{7}$ total cells per flask (Fig. S2). Flasks were incubated at $30{ }^{\circ} \mathrm{C}$ with shaking at $150 \mathrm{rpm}$. At 0 and $64 \mathrm{~h}$, an aliquot of culture was removed from each flask for CFU enumeration on PYE agar plates (Fig. S3). After $\sim 64 \mathrm{~h}$ of growth, cells from all three flasks were collected by filtration using an Express Plus Membrane $0.22 \mu \mathrm{m}$ filter (Millipore). Filters were stored at $-80^{\circ} \mathrm{C}$ until needed. To mimic saturating conditions with the same number of doublings in defined M2X and complex PYE laboratory medium as in lake water, we inoculated cultures 
at a concentration that after five doublings (the estimated number of doublings in lake water), the cultures reached saturation. Cells were pelleted at $10,000 \times g$ for $1 \mathrm{~min}$ and stored at $-20{ }^{\circ} \mathrm{C}$. Genomic DNA from all samples was extracted using guanidium thiocyanate as previously described [19], with the exception that the lake water samples were lysed directly from the filters they were collected on. DNA quality and quantity was measured using a NanoDrop $^{\text {OneC }}$ (Thermo Scientific).

\section{Amplification and sequencing of Tn-Himar barcodes}

PCR amplification for each sample was performed as previously described [9] (Fig. S1) using a standard reaction protocol for Q5 DNA polymerase (New England BioLabs) with the primers BarSeq_P1 and 1 of 16 forward primers (BarSeq_P2_IT001 to BarSeq_P2_IT016; Table S1) containing unique 6-bp TruSeq indexes that were sequenced using a separate index primer. Cycling conditions were as follows: $98^{\circ} \mathrm{C}$ for $4 \mathrm{~min}$ followed by 25 cycles of $30 \mathrm{~s}$ at 98 ${ }^{\circ} \mathrm{C}, 30 \mathrm{~s}$ at $55^{\circ} \mathrm{C}$, and $30 \mathrm{~s}$ at $72^{\circ} \mathrm{C}$, followed by a final extension at $72^{\circ} \mathrm{C}$ for $5 \mathrm{~min}$. PCR products were purified using GeneJET PCR Purification Kit (Thermo Scientific). Purified samples were run on a $2.5 \%$ agarose gel to confirm correct product size ( $200 \mathrm{bp})$. A total of $10 \mu \mathrm{L}$ per purified PCR product was pooled, assessed for quality, and quantified using a Bioanalyzer. The amplified barcodes from the reference (PYE) and treatment (M2X, unfiltered lake water, and filtered lake water) were sequenced on an Illumina HiSeq4000 at the University of Chicago Genomics Facility, multiplexing all 16 samples in one lane with 50-bp singleend reads. All sequence data have been deposited in the NCBI Sequence Read Archive under BioProject accession PRJNA429486; BioSample accession SAMN08348121; SRA accession SRP128742.

\section{Analysis of Tn-Himar strain fitness}

We followed the fitness calculation protocol of Wetmore and colleagues [9], using scripts available at https:// bitbucket.org/berkeleylab/feba. Briefly, the total count of each barcode in each sample was calculated using a Perl script (MultiCodes.pl) and, from this table of barcodes, strain fitness was calculated using an $\mathrm{R}$ script (FEBA.R). The fitness of each strain was calculated as a normalized $\log _{2}$ ratio of barcode counts in the treatment sample to counts in the PYE reference sample. The fitness of genes was calculated as the weighted average of strain fitness values, the weight being inversely proportional to a variance metric based on the total number of reads for each strain; this weighting is fully described by Wetmore and colleagues [9]. Successful gene fitness calculations required at least 3 reads per strain and 30 reads for each of the
16 samples. Insertions in the first $10 \%$ or last $10 \%$ of a gene were not considered in gene fitness calculations. The complete dataset of fitness values for each condition is listed in Table S2.

To assess the distribution of fitness scores, we calculated the standard deviation for each condition using the frequency distribution of the mean fitness value of each gene (filtered lake water $=0.41$, unfiltered lake water $=0.40$, defined medium $=1.1)$. When the outlier region of the defined medium dataset $(<-2.5)$ was removed, the calculated standard deviation was 0.36 ; therefore, a standard deviation of 0.4 was chosen and applied to all conditions. Genes with a mean fitness value approximated at $\pm 3 \sigma$ from the mean $(<-1.2$ and $>+1.2)$ were selected for further examination. We also examined $t$-values, the fitness value of a gene divided by a variance metric, based on the total number of reads for each gene (as previously described [9]), to provide a metric to assess the significance of fitness values (Table S3).

To identify genes showing differential fitness across lake water samples, we fit a linear model with two factors, sampling day and filtration treatment (filtered or unfiltered). The model was implemented using the functions lmfit, eBayes, and topTable in the $\mathrm{R}$ package limma [23]. Genes were identified as having differential fitness across either sampling days or filtration treatment, with a false discovery rate threshold of 0.05 .

\section{Analysis of Caulobacter Tn5-seq fitness}

A Caulobacter Tn5 insertion library containing an estimated $3 \times 10^{5}$ clones was constructed as previously described [24]. The lake water fitness experiment for the Tn5 library (from Lake Michigan water collected in April 2016) was performed similarly to the Tn-Himar library experiments with the following modifications: a total of $200 \mu \mathrm{L}$ of the Caulobacter Tn5 library was inoculated into $20 \mathrm{~mL}$ of PYE for the initial outgrowth for $5 \mathrm{~h}$, which was then inoculated into $2 \mathrm{~L}$ for the PYE and unfiltered lake water treatments, and two replicates of $2 \mathrm{~L}$ each for filtered lake water. Lake water cultures were harvested by filtration after $60 \mathrm{~h}$ of growth, and the PYE condition was filtered after $12 \mathrm{~h}$ to approximate the same number of doublings. However, our PYE cultures achieved over six doublings, versus four doublings for lake water.

A nested PCR approach was used to specifically amplify transposon-containing DNA fragments for sequencing. A low cycle PCR amplification for each sample was first performed using a standard reaction protocol for KOD Xtreme $^{\mathrm{TM}}$ Hot Start Polymerase with 5\% dimethylsulfoxide (DMSO) and $0.3 \mu \mathrm{M}$ primer using the primers $\mathrm{F} 1$ and P7 [24] (Table S1). Cycling conditions were as follows: $95^{\circ} \mathrm{C}$ for $90 \mathrm{~s} ; 5$ cycles of $95^{\circ} \mathrm{C}$ for $15 \mathrm{~s}, 68^{\circ} \mathrm{C}$ for $30 \mathrm{~s}$, and $72{ }^{\circ} \mathrm{C}$ 

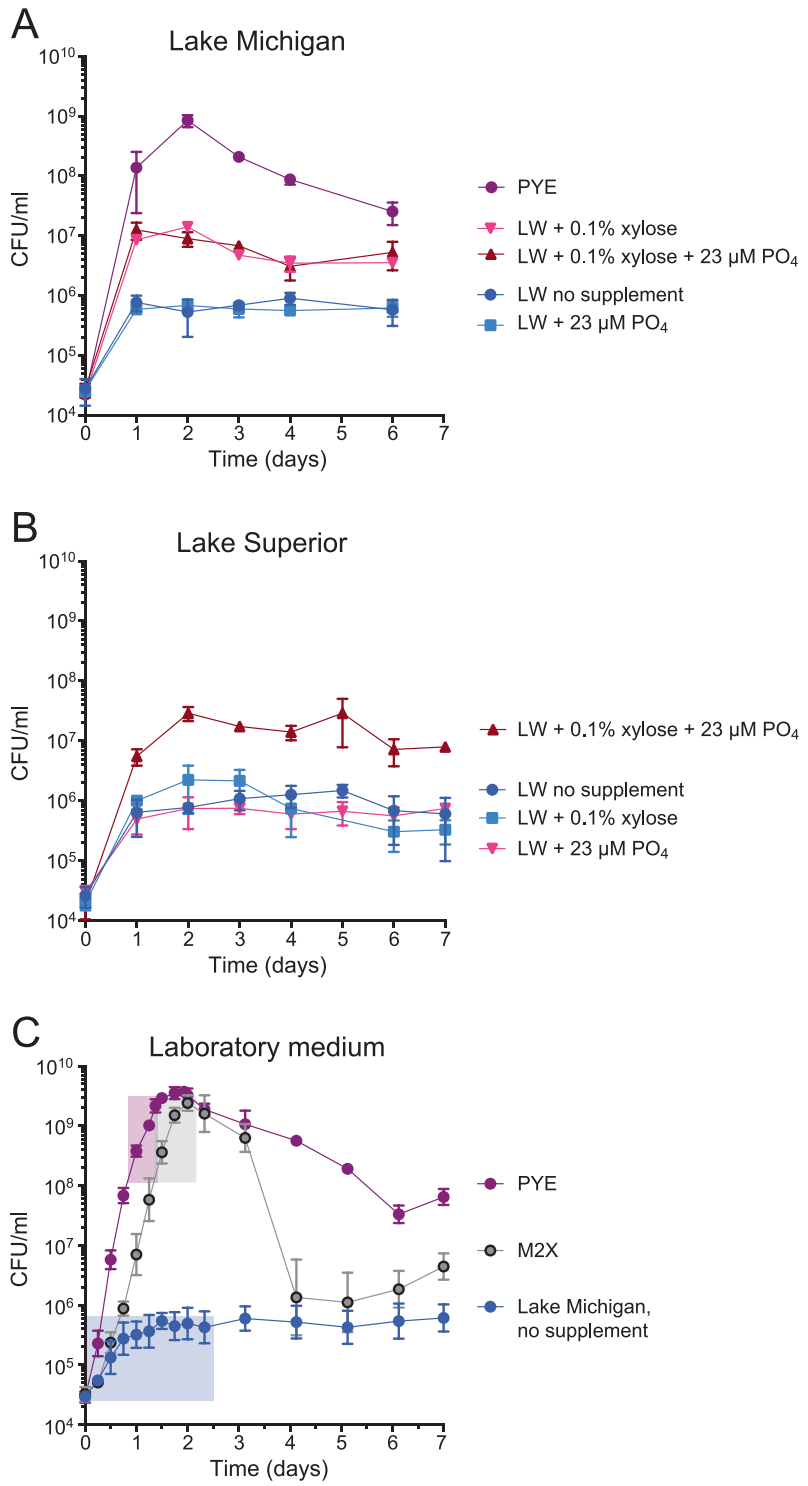

Fig. 1 Growth of Caulobacter in laboratory medium, and supplemented or unsupplemented water from two Great Lakes. Overnight cultures washed with filtered lake water (LW) were inoculated into 5 $\mathrm{mL}$ of filtered water from Lake Michigan (a) or Lake Superior (b). Growth assays in water supplemented with carbon $(0.1 \% \mathrm{w} / \mathrm{v}$ xylose $)$ and/or phosphorus $\left(23 \mu \mathrm{M} \mathrm{K}_{2} \mathrm{HPO}_{4}\right)$ as indicated; growth was monitored every $24 \mathrm{~h}$ by enumeration of colony-forming units (CFUs) by dilution plating. Lake water growth is compared with growth in a laboratory peptone yeast extract (PYE) medium. Data represent mean \pm standard deviation of five replicates per condition. c Fine scale growth of Caulobacter in PYE, M2-xylose defined medium (M2X), and filtered Lake Michigan water. Cells were grown as in $\mathbf{a}$ and $\mathbf{b}$ and monitored by enumerating CFUs. Data represent mean \pm standard deviation of five replicates per condition. Boxes represent the approximate region of the growth curve (cell density and incubation time) in which the barcoded Tn-Himar mutant library (BarSeq) pools were cultivated

for $30 \mathrm{~s} ; 13$ cycles of $95^{\circ} \mathrm{C} 15 \mathrm{~s}, 55^{\circ} \mathrm{C} 30 \mathrm{~s}$, and $72{ }^{\circ} \mathrm{C} 30 \mathrm{~s}$, followed by a final extension at $72{ }^{\circ} \mathrm{C}$ for $5 \mathrm{~min}$. Samples were treated with ExoSAP-ITTM PCR product cleanup reagent (Thermo Fisher Scientific) according to manufacturer's protocol. A second PCR step was performed with the transposon-specific primer containing the adapter sequence using KOD Xtreme ${ }^{\mathrm{TM}}$ Hot Start Polymerase with $5 \%$ DMSO and $0.3 \mu \mathrm{M}$ primer in a $62.5-\mu \mathrm{L}$ reaction volume using the primers Tn5-left and P7 [24] (Table S1). Cycling conditions were as follows: $95^{\circ} \mathrm{C}$ for $3 \mathrm{~min}, 12$ cycles of 95 ${ }^{\circ} \mathrm{C}$ for $30 \mathrm{~s}, 55^{\circ} \mathrm{C}$ for $30 \mathrm{~s}$, and $72{ }^{\circ} \mathrm{C}$ for $30 \mathrm{~s}$, followed by a final extension at $72{ }^{\circ} \mathrm{C}$ for $5 \mathrm{~min}$. Product size ( 200 bp) was confirmed on a $1 \%$ agarose gel. After standard bead cleanup and Illumina library preparation, samples were sequenced at the University of Chicago Genomics Facility using a custom sequencing primer [24] (Table S1).

Fitness analysis was performed as previously described [25] using the TRANSIT software (available at https:// github.com/mad-lab/transit). We used the permutation test in TRANSIT to quantify differences in sequencing read counts between our PYE and lake water conditions [25]. The complete Tn5 dataset (Tables S4 and S5), genes with differential fitness $(p<0.01$; Tables S5 and S6), and genes shared between the Tn5 and Tn-Himar datasets (Table S7) are listed in Supplemental Information. Raw Tn-Seq data are deposited in the NCBI sequence read archive under BioProject accession PRJNA429486; BioSample accession SAMN08348191; and SRA accession SRP128742.

\section{Results}

\section{Growth of Caulobacter in natural freshwater}

As a prerequisite to measuring strain fitness, we first sought to demonstrate Caulobacter growth in natural freshwater. We collected nearshore water from Lake Michigan, representing a typical freshwater system inhabited by Caulobacter spp. [26, 27]. With no additional supplementation, filtered $(0.1 \mu \mathrm{m})$ lake water supported Caulobacter growth to a maximal density of approximately $5 \times 10^{5} \mathrm{CFU} \mathrm{mL}^{-1}$ (Fig. 1a), from an initial inoculum of $2.5 \times 10^{4} \mathrm{CFU} \mathrm{mL}^{-1}$. Caulobacter doubled 4-5 times at a rate of $0.14 \mathrm{~h}^{-1}$ (doubling time $5 \mathrm{~h}$ ). Similar growth rates were observed in unfiltered lake water. Supplementation with $0.1 \%$ xylose increased the maximal density by about 10 -fold, whereas addition of $23 \mu \mathrm{M} \mathrm{K} \mathrm{K}_{2} \mathrm{HPO}_{4}$ had no effect (Fig. 1a), implying that carbon, but not phosphorus, limits Caulobacter growth in Lake Michigan water. For comparison, we also assayed Caulobacter growth in water collected from Lake Superior and found a similar growth yield (Fig. 1b). Supplementation with either $23 \mu \mathrm{M} \mathrm{K}_{2} \mathrm{HPO}_{4}$ or $0.1 \%$ xylose did not significantly enhance Caulobacter growth, but together $0.1 \%$ xylose and $23 \mu \mathrm{M} \mathrm{K}_{2} \mathrm{HPO}_{4}$ enhanced growth by more than 10 -fold, suggesting that both carbon and phosphorus limit growth in Lake Superior. By 
Table 2 Transposon library statistics

\begin{tabular}{llll}
\hline Library & Unique insertion sites & Average transposons per ORF & Mean reads \\
\hline Tn5 $^{\mathrm{a}}$ & 115,788 & 30 & $90-150($ per Tn) \\
Tn-Himar $^{\mathrm{b}}$ (BarSeq) & 46,395 & 24 & $7612($ per gene) \\
\hline
\end{tabular}

${ }^{\mathrm{a}} \mathrm{Tn} 5$ can insert into any dinucleotide site. We identified 115,788 insertion sites in an initial population of $\sim 300,000$ clones

${ }^{\mathrm{b}} \mathrm{Tn}$-Himar specifically inserts into TA dinucleotides

comparison, Caulobacter reached a density of $3 \times 10^{9} \mathrm{CFU}$ $\mathrm{mL}^{-1}$ in PYE broth or in defined M2X medium (Fig. 1c). Notably, cell density was stable for 1 week in lake water but declined by $2-3$ orders of magnitude after 2 days of cultivation in artificial media (Fig. 1). This finding is consistent with a report by Poindexter describing Caulobacter isolates that tolerated prolonged nutrient scarcity with little loss of viability [11]. Based on our results, we chose to perform our genetic analysis in unsupplemented water from Lake Michigan.

\section{A global Tn-Seq approach identifies Caulobacter mutants with altered fitness in lake water}

We sought to identify genes required for Caulobacter growth in natural freshwater, compared with defined M2X or complex PYE medium. To this end, we constructed mutant libraries (Table 2) using two different transposons: Tn5, which inserts randomly, and Tn-Himar, which inserts specifically at TA dinucleotides, which occur on average every 82 bp in the Caulobacter genome. Each transposon in the pool of Himar transposons contains a unique 20-bp barcode sequence, which is mapped once to a specific insertion site in the genome and thereafter can be quantified by simple amplicon sequencing [9] (see Fig. S1). Both transposon libraries were constructed by growing cells in PYE; hence insertions in genes essential for growth in PYE are not represented in either library.

We cultivated the Caulobacter Tn5 pool in PYE and filtered lake water $(0.1 \mu \mathrm{m})$. Although Tn5 is capable of insertion at almost every position in the genome, our Tn5 library had lower site saturation than our Tn-Himar library, which limited the statistical power to identify significant fitness effects $[25,28]$. We calculated mutant fitness and gene essentiality for all genes (Tables S5 and S6) and identified 55 genes for which Tn5 disruption significantly diminished or enhanced growth in lake water relative to PYE (adjusted $p$-value cutoff $<0.01$ ). Given the limited power of the Tn5 dataset, we focused our analyses on the Tn-Himar dataset, but include the Tn5 data in the supplemental material as they provide useful validation of the TnHimar data discussed hereforward.

The Caulobacter Tn-Himar library contained an estimated $2 \times 10^{6}$ clones, of which $7 \times 10^{4}$ passed the criteria for barcode mapping [9]. Considering there are only $\sim 5 \times$ $10^{4} \mathrm{TA}$ insertion sites in the Caulobacter genome, it is clear that in this population we hit some sites more than once with unique barcodes. We cultivated this library in four conditions: (1) complex PYE medium, (2) defined M2X medium, (3) filtered lake water, and (4) unfiltered lake water (Fig. S2). To ensure that we started the experiment with sufficient mutant strain diversity, we inoculated the same total number of cells $\left(2.5 \times 10^{7}\right)$ in each treatment and aimed for 4-5 doublings into the late exponential phase of growth (Fig. 1 and S2). For PYE and M2X treatments, cells were grown in $1.5 \mathrm{~mL}$ volumes for 10 and $20 \mathrm{~h}$, respectively. For lake water treatments, cells were grown in three flasks each containing $2.5 \mathrm{~L}$ for $64 \mathrm{~h}$. By varying culture volume, we ensured an equal number of cell divisions across a similar phase of the growth curve. This approach required cultivation at different cell densities between conditions. After harvest, barcodes were analyzed as described [9], and strain fitness was calculated as the $\log _{2}$ of the ratio of barcode abundance in lake water (or M2X) to the control condition (PYE) [9]. Given the 20- to 30-fold increase in cell number of the mutant pool, a Tn-Himar insertion strain that did not grow at all should have a fitness score around -4 to -5 ; more extreme (lower) fitness scores indicate strains that did not survive cultivation. The most extreme negative fitness scores in this dataset (i.e., $<-4$ ) likely reflect genes that are essential in a particular condition (Figs. 2a, d; Table S2). The distributions of fitness scores in defined medium and the two lake water conditions are presented in Figs. 2b, c.

To validate our approach, we examined the fitness consequences of disrupting xylose utilization genes in the M2xylose (M2X) growth condition. Genes in the $x y l X A B C D$ operon are required for xylose utilization [29, 30]. As expected, insertions in these genes generated fitness scores of -3.6 to -6.6 when the pool was cultivated in M2X (Fig. 3a, Table S2). Disruption of $x y l R$, which functions as a transcriptional repressor of the xylose operon [29], resulted in a positive fitness score in M2X relative to PYE, indicating that derepression of the xylose utilization genes is advantageous when xylose is the sole carbon source. Disruption of the $x y l X A B C D$ genes had little effect on fitness in lake water, which contains a range of carbon sources beyond xylose; disruption of $x y l R$ resulted in a modest 

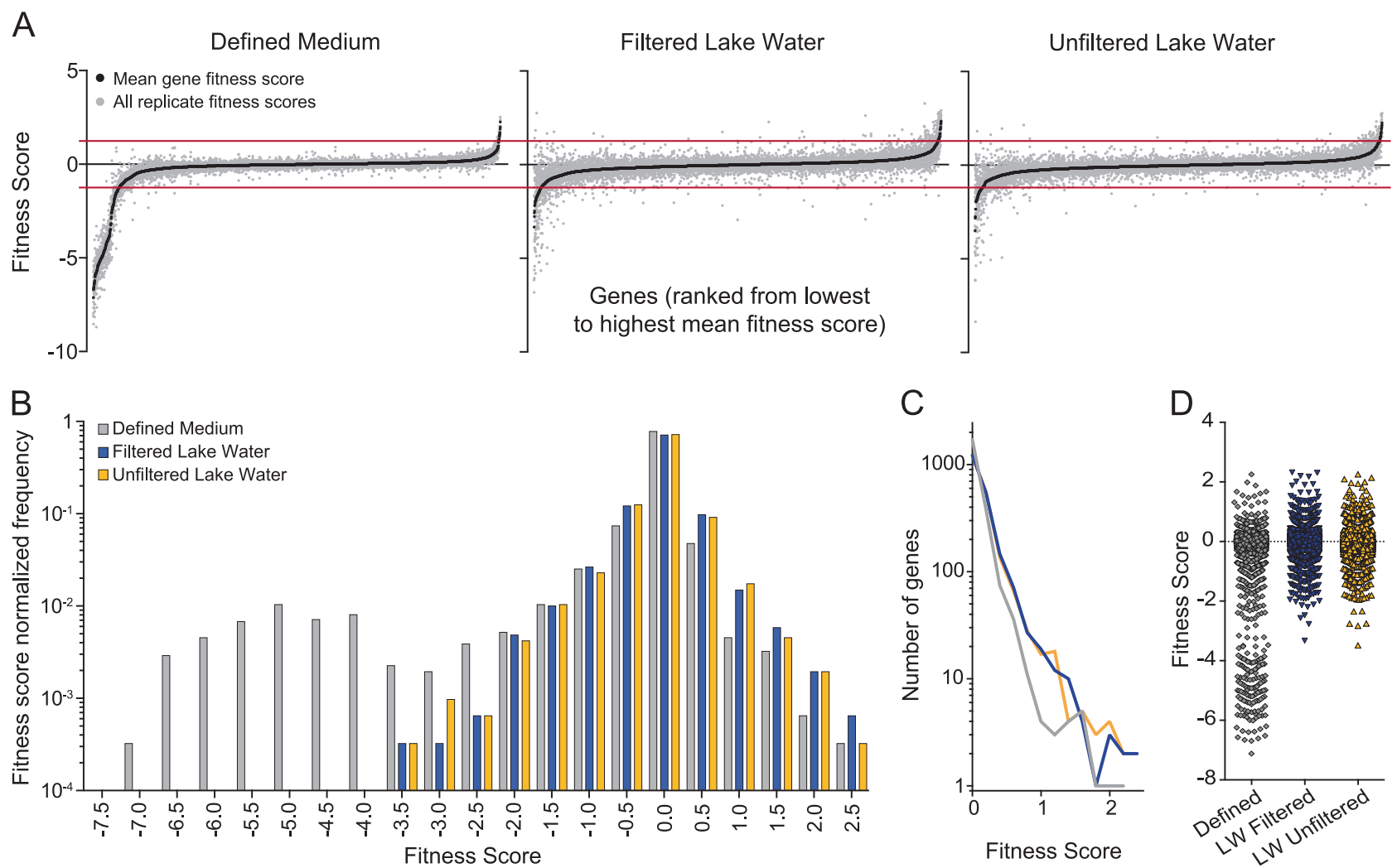

Fig. 2 Caulobacter gene fitness score summary after cultivation in defined medium, filtered, or unfiltered Lake Michigan water. a Rankordered mean fitness scores of each scorable Caulobacter gene across each of the four replicate experiments for each growth condition is plotted; black $=$ mean fitness score; gray $=$ independent replicate fitness scores. Red lines represent an estimate of $\pm 3 \sigma$ from the mean score of the entire dataset (which is approximately 0 ; genes with fitness scores $<-2.5$ in M2X were excluded from this determination). b Distributions of mean gene fitness scores for each condition: defined

fitness decrease in lake water relative to PYE (Fig. 3a), suggesting a cost to constitutive expression of unused genes.

\section{Increased variability of fitness scores in lake water}

Compared with defined M2X medium, lake water is more heterogeneous over time and space. Our four lake water experiments used water collected on 4 days over a 2-week period and showed greater variability in strain fitness scores than our four independent M2X replicates (Figs. 2a, 3b, 4). In addition, we fit a linear model to test the effects of two factors, sampling day and filtration condition, on strain fitness scores, and found a number of genes that differed significantly across days, including genes related to cell surface carbohydrate biosynthesis and environmental sensing and gene regulation (Table S8). This variability likely reflects day-to-day differences in temperature, mixing, and biotic factors such as phage dynamics, though we cannot completely rule out technical day-to-day variations in
M2X medium (gray), filtered Lake Michigan water (blue), and unfiltered Lake Michigan water (yellow). c Distribution of mean gene fitness values between 0 and +2.5 plotted for each condition; defined M2X medium (gray), filtered Lake Michigan water (blue), and unfiltered Lake Michigan water (yellow). d Gene fitness score distribution plotted for each of the three cultivation conditions: defined M2X medium (gray), filtered Lake Michigan water (blue), and unfiltered Lake Michigan water (yellow)

sample processing. Future work with additional temporal replicates could discriminate genes whose functions are consistently important from genes that are exploited under transient conditions in the lake.

Surprisingly, filtration $(0.1 \mu \mathrm{m})$ had little effect on the global fitness profile of Caulobacter (Fig. 4), and our linear model approach did not identify any genes with differential fitness between filtered and unfiltered lake water. This result implies that particulates and other microorganisms present in the lake water did not affect strain growth, suggesting that Caulobacter is not in strong competition with other microbes for "common goods" in this system on the timescale of our experiment.

\section{Fitness defects are more extreme in defined medium than in lake water}

Transposon disruption of genes required for amino-acid biosynthesis, nucleotide biosynthesis, lipopolysaccharide biosynthesis, and nucleotide sugar biosynthesis resulted in 
A
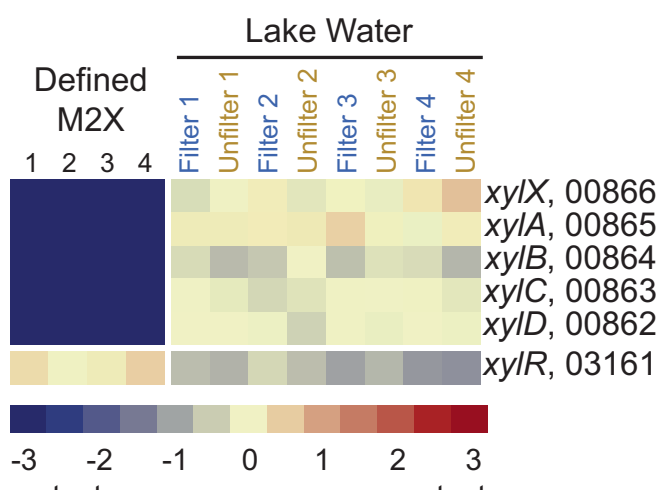

mutant

decreased

fitness

increased

fitness

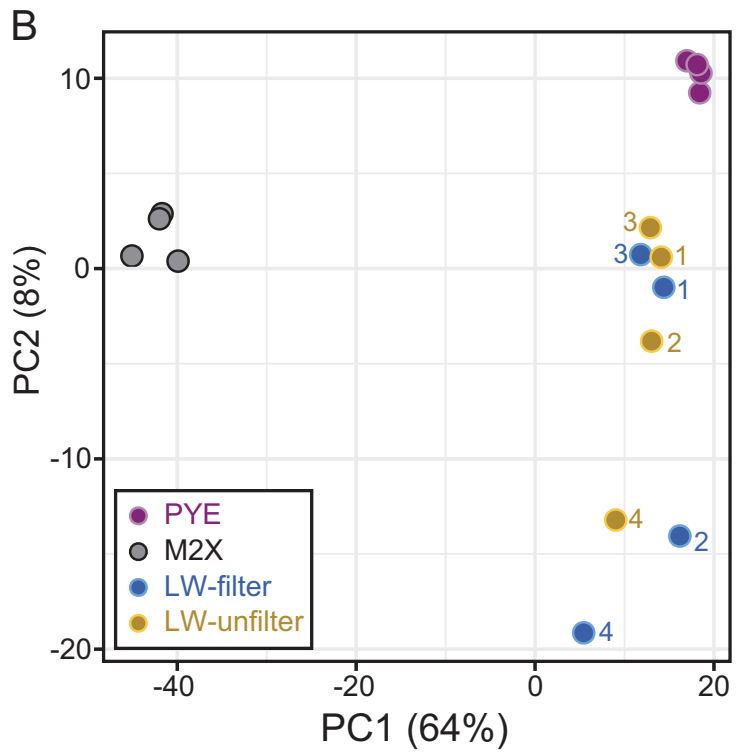

Fig. 3 Functional validation of the barcoded Tn-Seq approach; PCA profile of Tn strain fitness in complex medium, minimal defined medium and Lake Michigan water. a Heatmap of gene fitness scores for six xylose utilization genes $(x y l X, x y l A, x y l B, x y l C, x y l D, x y l R$, shown with corresponding gene locus numbers) from each replicate experiment of cultivation of the Tn-Himar mutant library in M2X defined medium, filtered lake water, and unfiltered lake water. Fitness score color scale bar is shown below the panel. b Principal component (PC) analysis (PCA carried out in ClustVis [49]) of genomic-scale fitness values for the barcoded Caulobacter Tn-Himar mutant library cultivated in complex peptone yeast extract (PYE) medium (reference set), defined M2X medium, filtered Lake Michigan water, and unfiltered Lake Michigan water. The plot shows PCA values for individual samples from each cultivation condition. Percentage of variance contributed by the first two PCs is noted on the axes. PCA plot is based on all fitness score values for all genes in the Tn-Himar datasets (see Table S2)

extreme (fitness score < -4) growth defects in M2X (Fig. 3, Tables S2-S3); these fitness scores provide evidence that strains harboring disruptions of these genes did not grow at all in M2X and thus likely comprise a strain/gene set that is essential in this defined condition. This result is not surprising, considering that growth in $\mathrm{M} 2 \mathrm{X}$ medium requires

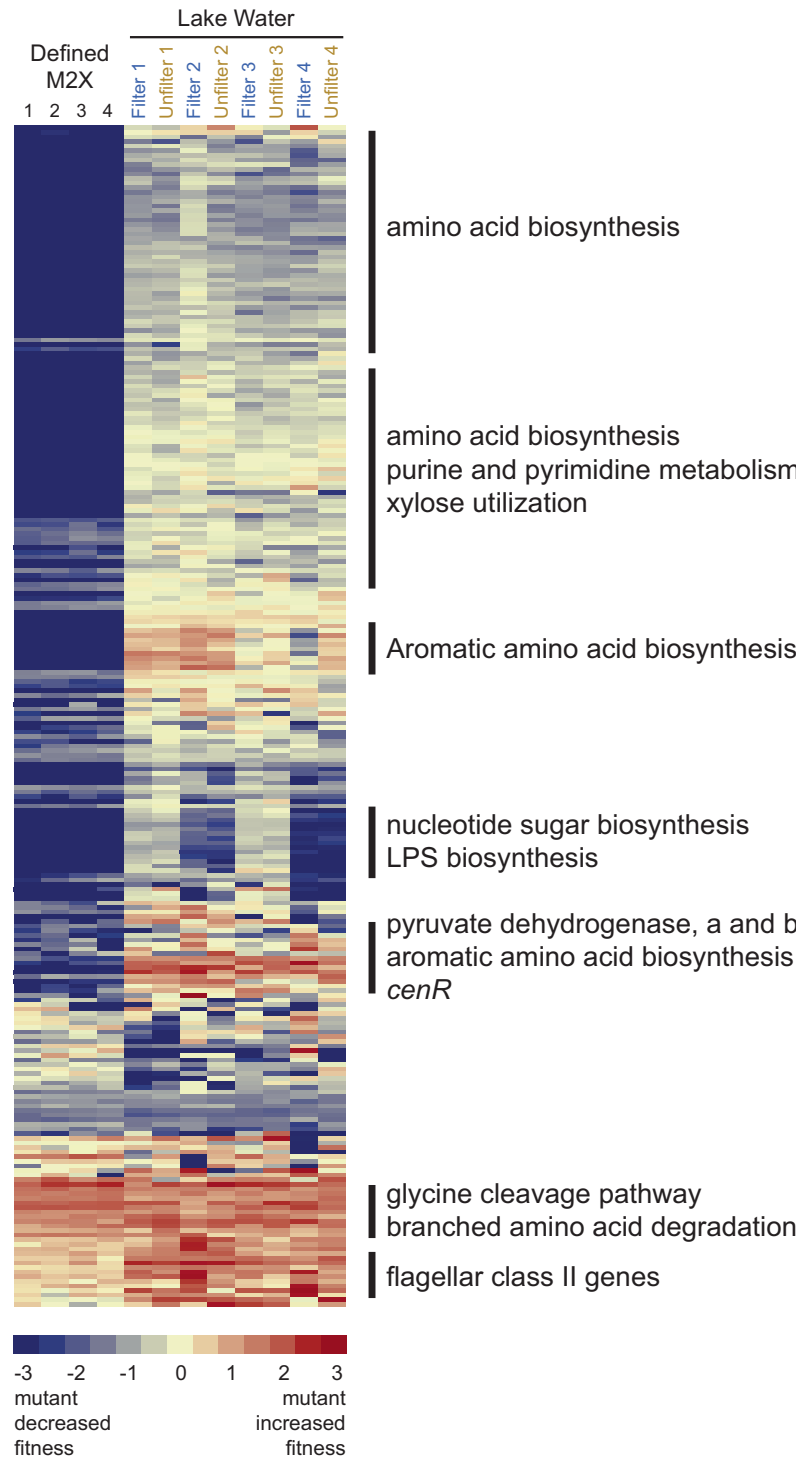

Fig. 4 Functional summary of mutant strains with diminished or enhanced fitness in minimal defined medium and Lake Michigan water. Heatmap of fitness scores for genes with mean fitness scores higher than 1.2 or lower than -1.2 (approximates $\pm 3 \sigma$ fitness score cutoff) in at least one cultivation condition. The sigma cutoff was based on the major fitness score distribution centered on zero (see Fig. 2b). Genes were hierarchically clustered using Cluster 3.0 (average linkage) and visualized using TreeView, and fitness scores for each replicate experiment are color coded on scale bar shown below. General functions of genes within particular regions of this 256 gene cluster are noted; this entire figure is expanded and split into three fully annotated clusters in Fig. S5, with gene names included. The full cluster of genes with mean fitness scores higher than +1.2 or lower than -1.2 in the lake water conditions (excluding the M2X data) is presented in Fig. S6

de novo biosynthesis of diverse monomers and intermediates, many of which are supplied exogenously in the reference PYE condition. In many cases, strains with severe fitness defects in M2X also had reduced growth in lake water, but the fitness costs were less severe (Tables S2 and S9-S10). We controlled the number of doublings 


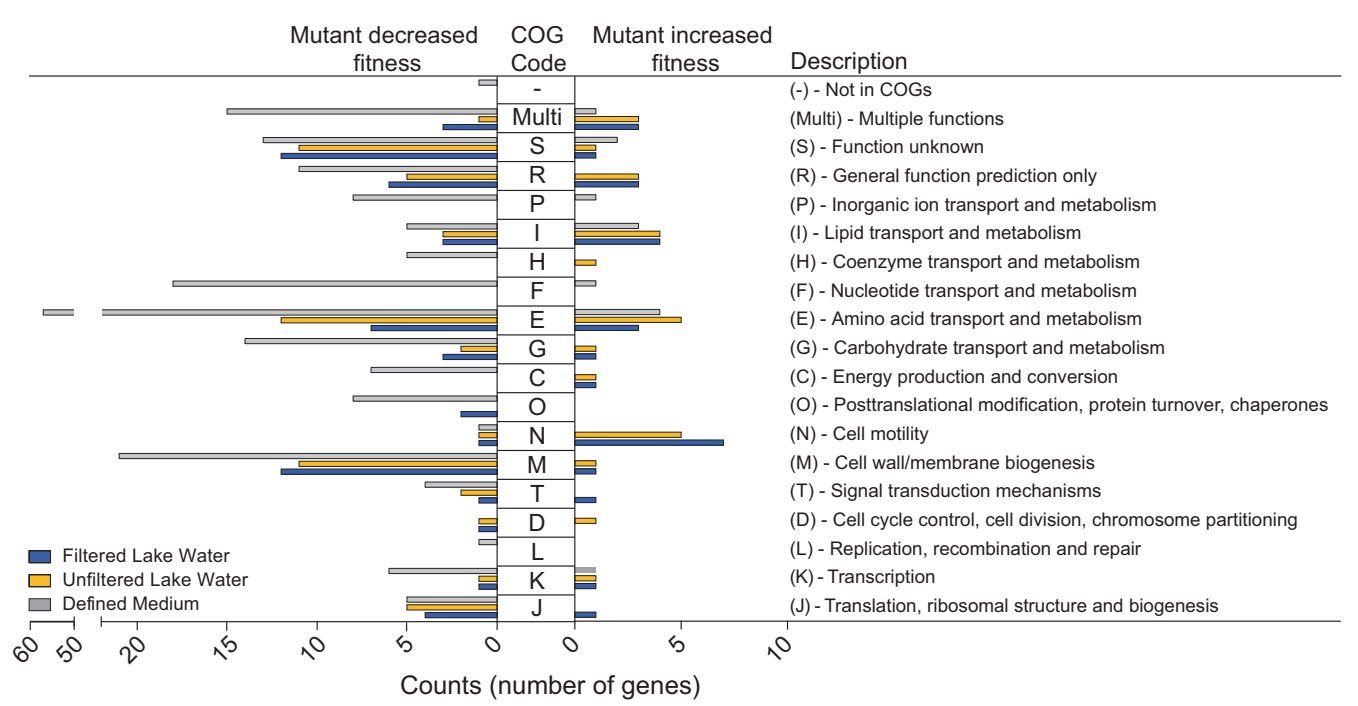

Fig. 5 COG analysis of Tn-Himar gene fitness data. The analysis includes genes with fitness scores of absolute value $>1.2$ (which approximates $a \pm 3 \sigma$ fitness score cutoff) in each condition. Each gene was assigned a cluster of orthologous group (COG) functional category, obtained through the NCBI COG site [31]. The number of genes in each COG category is plotted; genes with negative fitness values (left) and genes with positive fitness values (right)

(approximately 4-5) across all conditions, so the more pronounced fitness costs in defined medium compared with lake water cannot be explained by differences in the number of doublings. Instead, these results imply that lake water is more similar to the reference condition PYE than M2X is to PYE, in terms of the metabolic demands it imposes on cells. This inference is supported by principal component analysis across all growth conditions (Fig. 3b). Indeed, we expect that natural freshwater supplies diverse metabolites and growth substrates that may render some genes dispensable, whereas defined media provides fewer exogenous resources.

\section{Pathways conferring differential fitness in natural freshwater and artificial media}

To further explore the selective pressures faced by Caulobacter across these conditions, we focused on genes whose disruption induced large fitness effects, namely fitness scores $<-1.2$ and $>+1.2$ (this approximates a $\pm 3 \sigma$ cutoff). Based on this criterion, we identified 83 and 82 genes in the filtered and unfiltered lake water conditions, respectively, and 213 genes in the defined M2X medium (Table S9). Genes with significant fitness values across all three conditions based on the $t$-statistic of Wetmore and colleagues [9] are outlined in Table S10. Broad functional patterns in our Tn-Himar dataset were assessed using clusters of orthologous group (COG) annotations [31] (Fig. 5). A full comparison of genes for which Tn-Himar disruption results in a specific advantage or disadvantage in M2X defined medium, but not in filtered or unfiltered Lake Michigan water (relative to complex PYE medium), and vice versa, are presented in Tables S11-S12. Genes that were not hit by Tn-Himar, and thus not included in any of our analyses are included in Table S13. Many of these genes have been previously defined as essential [18]. A clustered heatmap that contains genes with fitness scores $<-1.2$ and $>+1.2$ from either the filtered or unfiltered lake water conditions is presented in Fig. S6.

Not surprisingly, the most negative fitness scores were observed for genes in amino-acid and nucleotide biosynthesis (discussed above), and in genes required for transport of metabolic substrates into the cell (Tables S2 and S9); we observed similar defects in our Tn5 dataset (Tables S4-S6). In addition, disruption of genes encoding catabolic enzymes in the glycine cleavage pathway and in branched aminoacid degradation led to an apparent enhancement of fitness in both M2X medium and in lake water relative to PYE, in both the Tn-Himar and Tn5 experiments (Fig. 4; Tables S2 and S4-S7). This result likely reflects the higher cost of deleting these catabolic genes in the reference PYE condition compared with $\mathrm{M} 2 \mathrm{X}$ or lake water, and is consistent with transcriptional data showing that select amino-acid degradation pathways-including glycine cleavage, histidine, branched chain, and phenylalanine degradation-are upregulated in PYE compared with M2X [32].

Surprisingly, we found enhanced fitness for strains with disruptions in motility genes in lake water relative to PYE (Figs 4 and 5). We more carefully examined the fitness scores of genes involved in synthesis and assembly of the flagellum (Fig. 6). The flagellum is assembled in a regulated hierarchy of stages, which is well described in Caulobacter [33-35]. Class II genes encode the inner components of the flagellum, including the export apparatus, and regulatory proteins that 


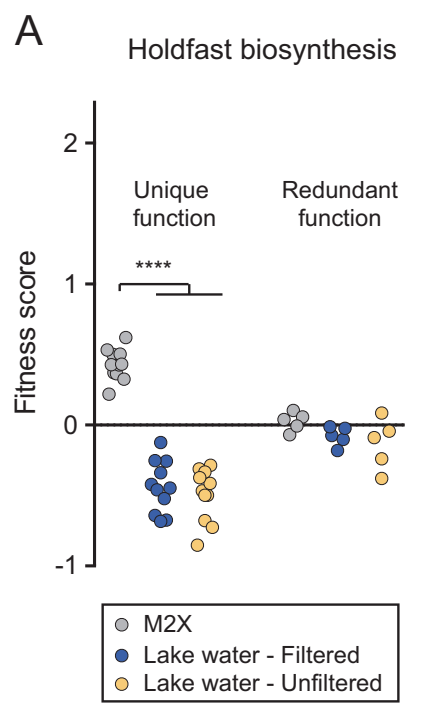

Fig. 6 Genes with functions in flagellum and holdfast biosynthesis influence fitness in Lake Michigan water. Mean fitness scores of genes involved in a holdfast biosynthesis (17 genes) and b flagellum biosynthesis (38 genes) are plotted for each cultivation condition (four independent growth replicates): defined M2X medium (gray); filtered lake water (blue); and unfiltered lake water (yellow). Holdfast genes with unique function (i.e., single gene deletions have a holdfast defect)

activate expression of class III and IV genes. Class III genes encode the basal body and hook structures. Completion of class III structures activates translation of class IV genes, which encode the subunits of the flagellar filament. Thus, defects in each class prevent expression of subsequent classes. Within each class of flagellar genes, we observed consistent fitness patterns, demonstrating the power of this method to capture even modest effects of gene disruption. Disruption of class II flagellar genes conferred an advantage that was significantly greater in lake water than in M2X compared with PYE (Fig. 6b and S2). Disruption of class III genes followed similar trends, but with smaller magnitude effects. Caulobacter encodes six redundant class IV flagellin genes [36], three of which are represented in our Tn-Himar pool and whose disruption, not surprisingly, had no effect on fitness. Disruption of the motor stator genes motA or $\operatorname{mot} B$, which results in a fully assembled but paralyzed flagellum [37, 38], did not affect fitness under our cultivation conditions. Together, these results suggest that the fitness advantage of flagellar gene disruption is not derived from energy saved in powering the flagellum, but rather in energy or resources saved in synthesizing and assembling the flagellum. In the lake water cultivations, we observed appreciable day-to-day variation in the fitness of each class of flagellar gene mutants (Fig. S2), which was particularly pronounced for class III genes. Patterns in this day-to-day variability were consistent across members of each class, suggesting that this variability is driven by environmental factors rather than technical factors.

Fitness was also affected by the ability to synthesize the polar extracellular adhesin known as the holdfast (Fig. 6a and redundant function (i.e., single mutants have no holdfast defect) are shown separately. Class II, Class III, and Class IV genes in the flagellar hierarchy and $m o t A / m o t B$ stator complex genes are also shown separately. Clusters of holdfast and flagellum fitness score data, with individually annotated genes, are shown in Fig. S4. One-way ANOVA was applied to assess differences in fitness scores between marked groups; Tukey's post test $(* * * * p<0.0001 ; * p<0.05)$

and S2). We systematically analyzed genes involved in synthesis, secretion, and attachment of the holdfast. Most holdfast genes yield partial to complete defects in holdfast development when disrupted [39]; we categorized these genes as 'unique functions' genes. However, two sets of holdfast biosynthesis genes have redundant functions: two Wzy-family polymerase genes function in holdfast development and three paralogs of the HfsE glycosyltransferase have genetically redundant activities in holdfast synthesis [39]. Disruption of genes in these redundant sets had no effect on fitness. Disruption of genes in the unique function group resulted in a modest but consistent fitness advantage in M2X and a fitness disadvantage in both filtered and unfiltered lake water, relative to PYE (Fig. 6a and S2A). For the group of all unique genes, the fitness consequence for loss of holdfast was significantly different between growth conditions $(p<0.0001)$ (Fig. 6a).

\section{Genes of unknown function contribute to fitness in natural freshwater}

We hypothesized that many genes of unknown function play important roles in natural environmental contexts but not in typical laboratory media. Of all genes showing large fitness effects $( \pm 3 \sigma)$, hypothetical genes or genes of unknown function accounted for $16 \%(13 / 83)$ in filtered lake water, $15 \%(12 / 82)$ in unfiltered lake water, and $7 \%$ in defined medium (15/213) (Table 3). Across these three conditions, five hypothetical genes were shared. CCNA_03860 was the only hypothetical gene for which 
Table 3 Average fitness scores for hypothetical genes and genes of unknown function with fitness scores $<-1.2$ and $>+$ 1.2 (bold) in at least one condition

\begin{tabular}{|c|c|c|c|c|}
\hline \multicolumn{2}{|l|}{ Locus number } & \multirow{2}{*}{$\frac{\text { Defined }}{-\mathbf{1 . 2 1}}$} & \multirow{2}{*}{$\frac{\text { Filtered LW }}{-\mathbf{2 . 5 7}}$} & \multirow{2}{*}{$\begin{array}{l}\text { Unfiltered LW } \\
\mathbf{- 2 . 8 3}\end{array}$} \\
\hline CCNA_00375 & No conserved domains & & & \\
\hline CCNA_01724 & COG4649, TPR_21 pfam09976 & -2.58 & -1.25 & -1.28 \\
\hline CCNA_03860 & COG3786, YkuD superfamily & 1.33 & 1.53 & 1.41 \\
\hline CCNA_03864 & DUF3576, pfam12100 & -1.71 & -1.32 & -1.57 \\
\hline CCNA_03909 & No conserved domains & -1.20 & -1.76 & -1.84 \\
\hline CCNA_00927 & No conserved domains & -2.56 & -2.11 & -0.67 \\
\hline CCNA_02875 & No conserved domains & -1.54 & -2.19 & -0.39 \\
\hline CCNA_00895 & No conserved domains & -0.19 & -2.14 & -1.44 \\
\hline CCNA_00913 & No conserved domains & -0.28 & -2.48 & -1.21 \\
\hline CCNA_00519 & No conserved domains & -1.66 & -0.37 & -0.61 \\
\hline CCNA_01176 & DUF2849, pfam11011 & -4.98 & -0.81 & -0.84 \\
\hline CCNA_01178 & DUF934, pfam06073, COG3749 & -4.36 & -0.60 & -0.64 \\
\hline CCNA_01676 & TamB, COG2911, pfam04357 & -6.40 & -0.52 & -0.74 \\
\hline CCNA_01219 & No conserved domains & 1.68 & -0.70 & 0.78 \\
\hline CCNA_02669 & $\begin{array}{l}\text { Uncharacterized membrane protein, } \\
\text { DUF3422, pfam11902, COG4949 }\end{array}$ & -4.41 & 0.38 & 0.20 \\
\hline CCNA_03273 & $\begin{array}{l}\text { COG4944, DUF1109, pfam06532 } \\
\text { NrsF, anti-sigF }\end{array}$ & -1.63 & -0.39 & -0.27 \\
\hline CCNA_03692 & COG1975, XdhC/CoxF family & -4.06 & -0.98 & -0.65 \\
\hline CCNA_02796 & No conserved domains & -0.45 & -1.20 & -0.52 \\
\hline CCNA_03420 & No conserved domains & 0.40 & -1.46 & 0.42 \\
\hline CCNA_03883 & No conserved domains & 0.05 & -1.25 & 0.99 \\
\hline CCNA_03984 & No conserved domains & 0.01 & -1.22 & -0.25 \\
\hline CCNA_01286 & No conserved domains & 0.25 & -0.25 & -1.27 \\
\hline CCNA_02053 & No conserved domains & -0.56 & -0.74 & -1.34 \\
\hline CCNA_02160 & No conserved domains & 0.26 & -1.02 & -1.38 \\
\hline CCNA_03015 & No conserved domains & -0.15 & 0.57 & -1.46 \\
\hline CCNA_03945 & No conserved domains & -0.43 & 0.24 & -1.90 \\
\hline
\end{tabular}

LW, Lake Michigan water disruption provided a fitness benefit across all three conditions relative to PYE. CCNA_03860 contains a conserved domain belonging to the YkuD superfamily, which has been shown to have L,D-transpeptidase catalytic activity, providing an alternate pathway for peptidoglycan cross-linking [40, 41]. Disruption of CCNA_01724, CCNA_03864,CCNA_03909, and CCNA_00375 resulted in reduced fitness across all three conditions relative to PYE. Hence using natural growth conditions may be critical for understanding the functions of many uncharacterized bacterial genes.

\section{Discussion}

\section{Tn-Seq fitness scores provide a window into cell- environment interactions}

Bacterial genomes carry relatively little noncoding DNA. Genes that confer no fitness benefit tend to decay over time [42] implying that genes that are maintained are beneficial at least under some circumstances. Yet traditional microbial cultivation approaches often fail to yield discernable mutant phenotypes for many genes. One approach to overcome this challenge is to interrogate gene function in more relevant ecosystem contexts, embracing physicochemical complexity. The genome-scale fitness analysis of Caulobacter transposon mutants reported in this study provides new understanding of genes that affect growth in a bona fide freshwater environment. Disruption of genes involved in biosynthesis of non-aromatic amino acids, lipopolysaccharides, and nucleotide sugars results in large fitness defects in natural freshwater compared with complex laboratory medium (PYE). Moreover, fitness effects were variable across temporal lake water replicates; this variability likely reflects physicochemical and biological variability in the lake and suggests an important role for transient response genes in fluctuating environments. 


\section{The fitness costs and benefits of motility and attachment in freshwater}

The energetic cost of flagellar biosynthesis and motility is well established [43, 44]. Our data indicate that transposon disruption of genes required for the synthesis of the single polar flagellum of Caulobacter enhanced fitness in lake water relative to PYE medium (Fig. 6b). This is consistent with a Salmonella Tn-Seq study that revealed a fitness advantage in strains with disrupted flagellar genes [45]. Notably, we found that fitness effects were not uniform across all flagellar genes: disruption of class II genes, which has the greatest impact on flagellar gene expression, also led to greater effects on fitness, compared with class III and IV genes. The fitness enhancement in lake water is not due to the energy savings from motor rotation, as strains with insertions in the motA and motB stator genes, which assemble a full but non-rotary flagellum [38], showed no fitness difference (Fig. 6b). We conclude that the relative fitness advantage of flagellar gene disruption is related to the cost of biosynthesis of flagellar proteins. It seems certain that over longer cultivation timescales, and in more spatially complex environments, the Caulobacter flagellum provides a fitness advantage, as flagellar genes are maintained in natural freshwater environments.

Our data reveal that disruption of genes required for holdfast biosynthesis is disadvantageous when strains are cultivated in lake water relative to PYE. This fitness cost was evident in both filtered (particle-free) and unfiltered lake water relative to PYE (Fig. 6a), suggesting that the effect is not due to adhesion to particles in the medium. Instead, it is possible that the holdfast confers a growth advantage by enabling adherence to the flask surface, where polymeric nutrients concentrate to form conditioning films [46, 47]. In defined M2X medium, disruption of holdfast biosynthesis genes confers a fitness advantage (Fig. 6a). In this medium, all the components are salts or simple sugars, which do not efficiently condition naive surfaces [46, 47]. In this case, surface attachment is apparently not advantageous, and holdfast biosynthesis comes at a cost.

\section{Genetic evidence suggests a complex medium is a better freshwater analog than a defined mineral medium}

Fitness defects of Caulobacter mutants were often more severe in a defined mineral xylose medium (M2X) than in lake water, relative to PYE. Moreover, the overall fitness profile of Caulobacter mutants cultivated in lake water more closely resembles that in PYE than in M2X, suggesting that dilute complex medium is a better proxy for natural freshwater. Caulobacter belongs to a group of dimorphic prosthecate (i.e., stalked) alphaproteobacteria that are often specialized for oligotrophic, dilute environments [10, 11]. Indeed, the inhibition of growth and stalk development due to excess nutrients was the first physiological property of $\mathrm{Cau}$ lobacter spp. to be described [48]. Complex and defined media of varying compositions have been outlined for cultivation of Caulobacter and related genera, but it is notable that dilute peptone $(<0.2 \% \mathrm{w} / \mathrm{v})$ generally supports growth of all dimorphic prosthecate bacteria [11]. This observation supports the notion that the natural nutrient environment of this class of bacteria is best captured by cultivation in a dilute complex medium that contains amino acids and other trace complex biomolecular components. Our data also demonstrate that an M2-based medium exerts highly specific metabolic constraints and is likely not an ecologically or physiologically relevant growth condition.

\section{An approach to study gene function in ecosystem context}

The explosion of bacterial genome sequence information has far outpaced our ability to characterize gene function using traditional approaches, leading to the accumulation of thousands of 'unknown' protein families. Many of these families are conserved throughout the bacterial domain, which is evidence that they confer a selective benefit in particular conditions. This leads to the following question: under what circumstances do these conserved families provide a fitness advantage? At the onset of this study, we hypothesized that many of these unknown protein families would prove to be important in the natural ecological context of a bacterium. Among the genes whose disruption leads to the greatest fitness effects $( \pm 3 \sigma)$ in filtered lake water relative to PYE, approximately $15 \%$ are hypothetical or conserved genes of unknown function (Table 3, S8-S9). The approach we describe here indicates that these genes of unknown function play an important role in Caulobacter physiology in a natural freshwater environment. Going forward, one can take advantage of lake-specific growth phenotypes to begin to define the functions of these genes in an ecologically relevant context.

Acknowledgements This work was supported by UChicago BIG grant to SC and MLC, and NIGMS grant R01GM087353 to SC. KLH was supported by an NIH Ruth Kirschstein Postdoctoral Fellowship (F32 GM122242) and a Chicago Biomedical Consortium Postdoctoral Core Grant (FP064244-01-PR). LMRR was supported by the NIH Molecular and Cellular Biology Training Grant (T32 GM007183). PDC is supported an NSF CAREER award (1552647); he began the Tn5 library construction in the laboratory of Dr. Yves V. Brun at Indiana University. We thank the members of the Crosson laboratory for helpful discussions, Tom Ioerger (Texas A\&M) for assistance with TRANSIT, Adam Deutschbauer (University of California-Berkeley) for the E. coli APA752 strain, and David Hershey for construction of the $C$. crescentus CB15 Himar transposon library. We also thank Pieter Faber and Abhilasha Cheruku from the University of Chicago Genomics Facility for technical advice and helpful discussions, and 
Kasia Kluszczynska, Xiomy Alejandro-Navarreto, Lydia Varesio, and Olivia Stovicek for help with lake water collection.

\section{Compliance with ethical standards}

Conflict of interest The authors declare that they have no conflict of interest.

\section{References}

1. Cao M, Goodrich-Blair H. Ready or not: microbial adaptive responses in dynamic symbiosis environments. J Bacteriol. 2017;199:e00883-16.

2. van Gestel J, Vlamakis H, Kolter R. Division of labor in biofilms: the ecology of cell differentiation. Microbiol Spectr. 2015;3:MB0002-2014.

3. Vos M, Wolf AB, Jennings SJ, Kowalchuk GA. Micro-scale determinants of bacterial diversity in soil. FEMS Microbiol Rev. 2013;37:936-54.

4. Azam F, Malfatti F. Microbial structuring of marine ecosystems. Nat Rev Microbiol. 2007;5:782-91.

5. Stocker R. Marine microbes see a sea of gradients. Science. 2012;338:628-33.

6. van Opijnen T, Camilli A. Transposon insertion sequencing: a new tool for systems-level analysis of microorganisms. Nat Rev Microbiol. 2013;11:435-42.

7. Kwon YM, Ricke SC, Mandal RK. Transposon sequencing: methods and expanding applications. Appl Microbiol Biotechnol. 2016;100:31-43.

8. Price MN, Wetmore KM, Waters RJ, Callaghan M, Ray J, Liu H, et al. Mutant phenotypes for thousands of bacterial genes of unknown function. Nature. 2018;557:503-9.

9. Wetmore KM, Price MN, Waters RJ, Lamson JS, He J, Hoover $\mathrm{CA}$, et al. Rapid quantification of mutant fitness in diverse bacteria by sequencing randomly bar-coded transposons. mBio. 2015;6: e00306-15.

10. Poindexter JS. Biological properties and classification of the Caulobacter group. Bacteriol Rev. 1964;28:231-95.

11. Poindexter JS. Dimorphic prosthecate bacteria: the genera Caulobacter, Asticcacaulis, Hyphomicrobium, Pedomicrobium, Hyphomonas and Thiodendron. In: Dworkin M, Falkow S, Rosenberg E, Schleifer K-H, Stackebrandt E, editors. The prokaryotes: volume 5: Proteobacteria: alpha and beta subclasses. New York, NY: Springer New York; 2006. p. 72-90.

12. Wilhelm R. Following the terrestrial tracks of Caulobacter redefining the ecology of a reputed aquatic oligotroph. ISME J. 2018. https://doi.org/10.1038/s41396-018-0257-z.

13. Allen HL. Primary pproductivity, chemo-organotrophy, and nutritional interactions of epiphytic algae and bacteria on macrophytes in the littoral of a lake. Ecol Monogr. 1971;41: 97-127.

14. Ely B. Genetics of Caulobacter crescentus. Methods Enzymol. 1991;204:372-84.

15. Drever JI. The geochemistry of natural waters: surface and groundwater environments. Englewood Cliffs, NJ: Prentice Hall; 1997.

16. Brunberg AK, Nilsson E, Blomqvist P. Characteristics of oligotrophic hardwater lakes in a postglacial land-rise area in midSweden. Freshw Biol. 2002;47:1451-62.

17. Carlson RE. Trophic state index for lakes. Limnol Oceanogr. 1977;22:361-9.

18. Christen B, Abeliuk E, Collier JM, Kalogeraki VS, Passarelli B, Coller JA, et al. The essential genome of a bacterium. Mol Syst Biol. 2011;7:528
19. Pitcher DG, Saunders NA, Owen RJ. Rapid extraction of bacterial genomic DNA with guanidium thiocyanate. Lett Appl Microbiol. 1989;8:151-6.

20. Kent WJ. BLAT-the BLAST-like alignment tool. Genome Res. 2002;12:656-64.

21. Marks ME, Castro-Rojas CM, Teiling C, Du L, Kapatral V, Walunas TL, et al. The genetic basis of laboratory adaptation in Caulobacter crescentus. J Bacteriol. 2010;192:3678-88.

22. Schrader JM, Zhou B, Li GW, Lasker K, Childers WS, Williams $\mathrm{B}$, et al. The coding and noncoding architecture of the Caulobacter crescentus genome. PLoS Genet. 2014;10:e1004463.

23. Ritchie ME, Phipson B, Wu D, Hu Y, Law CW, Shi W, et al. Limma powers differential expression analyses for RNAsequencing and microarray studies. Nucleic Acids Res. 2015;43: e47.

24. Curtis PD, Brun YV. Identification of essential alphaproteobacterial genes reveals operational variability in conserved developmental and cell cycle systems. Mol Microbiol. 2014;93:713-35.

25. DeJesus MA, Ambadipudi C, Baker R, Sassetti C, Ioerger TR. TRANSIT-a software tool for Himar1 TnSeq analysis. PLoS Comput Biol. 2015;11:e1004401.

26. Lee PO, McLellan SL, Graham LE, Young EB. Invasive dreissenid mussels and benthic algae in Lake Michigan: characterizing effects on sediment bacterial communities. FEMS Microbiol Ecol. 2015;91:1-12.

27. McMillan L, Stout R. Occurrence of Sphaerotilus, Caulobacter, and Gallionella in Raw and treated water. J Am Water Works Assoc. 1977;69:171-3.

28. Chao MC, Abel S, Davis BM, Waldor MK. The design and analysis of transposon insertion sequencing experiments. Nat Rev Microbiol. 2016;14:119-28.

29. Stephens C, Christen B, Watanabe K, Fuchs T, Jenal U. Regulation of D-xylose metabolism in Caulobacter crescentus by a LacI-type repressor. J Bacteriol. 2007;189:8828-34.

30. Stephens C, Christen B, Fuchs T, Sundaram V, Watanabe K, Jenal U. Genetic analysis of a novel pathway for D-xylose metabolism in Caulobacter crescentus. J Bacteriol. 2007;189:2181-5.

31. Galperin MY, Makarova KS, Wolf YI, Koonin EV. Expanded microbial genome coverage and improved protein family annotation in the COG database. Nucleic Acids Res. 2015;43:D261-9.

32. Hottes AK, Meewan M, Yang D, Arana N, Romero P, McAdams $\mathrm{HH}$, et al. Transcriptional profiling of Caulobacter crescentus during growth on complex and minimal media. J Bacteriol. 2004;186:1448-61.

33. Ardissone S, Viollier PH. Interplay between flagellation and cell cycle control in Caulobacter. Curr Opin Microbiol. 2015;28:8392.

34. Benson $\mathrm{AK}, \mathrm{Wu} \mathrm{J}$, Newton $\mathrm{A}$. The role of FlbD in regulation of flagellar gene transcription in Caulobacter crescentus. Res Microbiol. 1994;145:420-30.

35. Xu H, Dingwall A, Shapiro L. Negative transcriptional regulation in the Caulobacter flagellar hierarchy. Proc Natl Acad Sci Usa. 1989;86:6656-60.

36. Faulds-Pain A, Birchall C, Aldridge C, Smith WD, Grimaldi G, Nakamura $\mathrm{S}$, et al. Flagellin redundancy in Caulobacter crescentus and its implications for flagellar filament assembly. J Bacteriol. 2011;193:2695-707.

37. Ely B, Croft RH, Gerardot CJ. Genetic mapping of genes required for motility in Caulobacter crescentus. Genetics. 1984;108:52332.

38. Johnson RC, Ely B. Analysis of nonmotile mutants of the dimorphic bacterium Caulobacter crescentus. J Bacteriol. 1979;137:627-34

39. Toh E, Kurtz HD Jr, Brun YV. Characterization of the Caulobacter crescentus holdfast polysaccharide biosynthesis pathway 
reveals significant redundancy in the initiating glycosyltransferase and polymerase steps. J Bacteriol. 2008;190:7219-31.

40. Kumar P, Kaushik A, Lloyd EP, Li SG, Mattoo R, Ammerman $\mathrm{NC}$, et al. Non-classical transpeptidases yield insight into new antibacterials. Nat Chem Biol. 2017;13:54-61.

41. Bielnicki J, Devedjiev Y, Derewenda U, Dauter Z, Joachimiak A, Derewenda ZS. B. subtilis ykuD protein at 2.0 A resolution: insights into the structure and function of a novel, ubiquitous family of bacterial enzymes. Proteins. 2006;62:144-51.

42. Mira A, Ochman H, Moran NA. Deletional bias and the evolution of bacterial genomes. Trends Genet. 2001;17:589-96.

43. Martinez-Garcia E, Nikel PI, Chavarria M, de Lorenzo V. The metabolic cost of flagellar motion in Pseudomonas putida KT2440. Environ Microbiol. 2014;16:291-303.

44. Smith DR, Chapman MR. Economical evolution: microbes reduce the synthetic cost of extracellular proteins. MBio. 2010. https:// doi.org/10.1128/mBio.00131-10.
45. Langridge GC, Phan MD, Turner DJ, Perkins TT, Parts L, Haase J, et al. Simultaneous assay of every Salmonella Typhi gene using one million transposon mutants. Genome Res. 2009;19: 2308-16.

46. Loeb GI, Neihof RA. Marine conditioning films. Applied Chemistry at Protein Interfaces. Adv Chem. 1975;145:319-35.

47. Schneider RP, Leis A. Conditioning films in aquatic environments. Encyclopedia of Environmental Microbiology. John Wiley \& Sons, Inc. New York, NY; 2003.

48. Loeffler F. Weitere Untersuchungen uber die Beizung und Farbung der Geisseln bei den Bakterien. Cent Bakteriol Parasitenkd. 1890;7:625-39.

49. Metsalu T, Vilo J. ClustVis: a web tool for visualizing clustering of multivariate data using Principal Component Analysis and heatmap. Nucleic Acids Res. 2015;43: W566-70. 